Images du travail, travail des images

6-7| 2019

Femmes au travail\&nbsp: quelles archives visuelles ?

\title{
Les métiers dits « féminins » dans les films de Jean Benoit-Lévy
}

Women's work in Jean Benoit-Lévy cinema

Pascal Laborderie

\section{OpenEdition}

Journals

Édition électronique

URL : http://journals.openedition.org/itti/719

DOI : $10.4000 /$ itti. 719

Éditeur

Université de Poitiers

\section{Référence électronique}

Pascal Laborderie, «Les métiers dits « féminins » dans les films de Jean Benoit-Lévy », Images du

travail, travail des images [En ligne], 6-7 | 2019, mis en ligne le 01 février 2019, consulté le 14 avril 2021 URL : http://journals.openedition.org/itti/719 ; DOI : https://doi.org/10.4000/itti.719

Ce document a été généré automatiquement le 14 avril 2021

Images du travail, travail des images 


\title{
Les métiers dits « féminins » dans les films de Jean Benoit-Lévy
}

Women's work in Jean Benoit-Lévy cinema

\author{
Pascal Laborderie
}

1 En France, dans l'entre-deux-guerres, la Ligue de l'enseignement, les Offices du cinéma éducateur qui lui étaient affiliés au sein de l'Union française des offices du cinéma éducateur laïque (UFOCEL) ${ }^{1}$, ainsi que leurs correspondants, principalement des établissements publics, instituèrent un vaste réseau d'éducation populaire par le cinéma : le cinéma scolaire et éducateur laïque. Tandis que les séances scolaires et périscolaires programmaient des films d'enseignement qui s'adressaient aux élèves dans le cadre de la classe (le cinéma scolaire), les activités postscolaires et populaires du cinéma éducateur formaient les jeunes gens et les adultes à la santé et la citoyenneté ainsi qu'à la vie économique, sociale et professionnelle.

2 Ayant réalisé et produit près de trois cent cinquante films, dont une trentaine de moyens et longs métrages, Jean Benoit-Lévy est sans nul doute le réalisateur le plus emblématique de ce cinéma. L'analyse de ses films a fait notamment l'objet de notre thèse, dans laquelle il est possible de trouver leur liste précise (Laborderie, 2009, 475-482) ${ }^{2}$. Ces films sont principalement conservés aux Archives françaises du film (AFF) du Centre national de la cinématographie et de l'image animée (CNC). Quelquesuns ont été numérisés et sont consultables à l'antenne du CNC de la Bibliothèque nationale de France.

3 Cette source audiovisuelle pour l'étude des représentations des métiers dits « féminins » est intéressante dans la mesure où ces films sont à la fois le vecteur et le reflet d'une idéologie dominante. En effet, Jean Benoit-Lévy peut être considéré comme le propagandiste "patenté » de la IIIe République (Vignaux, 2008, 205), parce qu'il promeut les valeurs républicaines ainsi que des principes élémentaires en matière d'hygiène. Cependant, cette propagande relativement consensuelle développe aussi des thématiques qui constituent le socle d'un discours laïque plus marqué politiquement à gauche, dont une vision progressiste de la place des femmes dans la société. 
Selon une double approche historique et sémiologique, notre analyse des films de Jean Benoit-Lévy montre qu'ils sont empreints des stéréotypes propres à leur époque, mais qu'ils témoignent aussi d'un regard évolutif sur les relations de travail entre les femmes et les hommes. En effet, en raison du fait que sa cinématographie s'étend sur plusieurs années, Jean Benoit-Lévy a pu changer sa manière de considérer le travail féminin. Dans cette perspective, nous aborderons les représentations des métiers des personnages féminins qui peuplent ses films, à partir d'un échantillon de courts métrages documentaires destinés à la classe (les films d'éducation à l'hygiène et les films d'orientation professionnelle) et des longs métrages réalisés pour le grand public, en particulier sur les métiers du soin dans La Future Maman (1924) et Le Voile sacré (1926) ainsi que sur les métiers de l'éducation dans La Maternelle (1932).

\section{Les courts métrages d'éducation hygiéniste et d'orientation professionnelle}

5 Si le cinéma de Jean Benoit-Lévy contribue à la construction des rapports genrés et mobilise les stéréotypes des métiers dits "féminins» et «masculins", dans quelle mesure ces productions à teneur idéologique sont-elles voulues ou inconscientes? En d'autres termes, comment distinguer l'implicite non intentionnel d'un film (celui qui participe à la reproduction involontaire d'un système), de l'implicite qui revêt quant à lui une dimension critique assumée par l'auteur? Selon nous, il est nécessaire, d'une façon complémentaire, d'une part, de mener l'étude d'un corpus, en effectuant un travail de sérialisation qui montre la récurrence d'un discours sur les rapports genrés, et d'autre part, de proposer une mise en perspective historique, en confrontant les représentations véhiculées par les films avec les données sociohistoriques dont nous disposons. D'une manière générale, l'analyse sérielle des films de Jean Benoit-Lévy révèle que les femmes y occupent une place considérable, dans le contexte du cinéma français des années 1930, où les personnages masculins sont hégémoniques (Sellier, 1999). Ainsi les jeunes femmes ont-elles presque toujours le rôle principal aussi bien dans ses courts que dans ses longs métrages (Flitterman-Lewis, 1990 ; Vignaux, 2007 ; Laborderie, 2009).

Images 1 et 2.

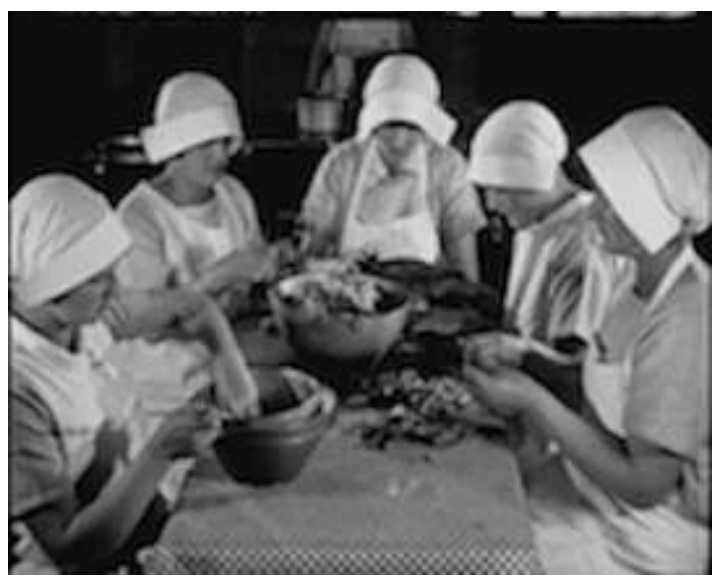


Image 2.

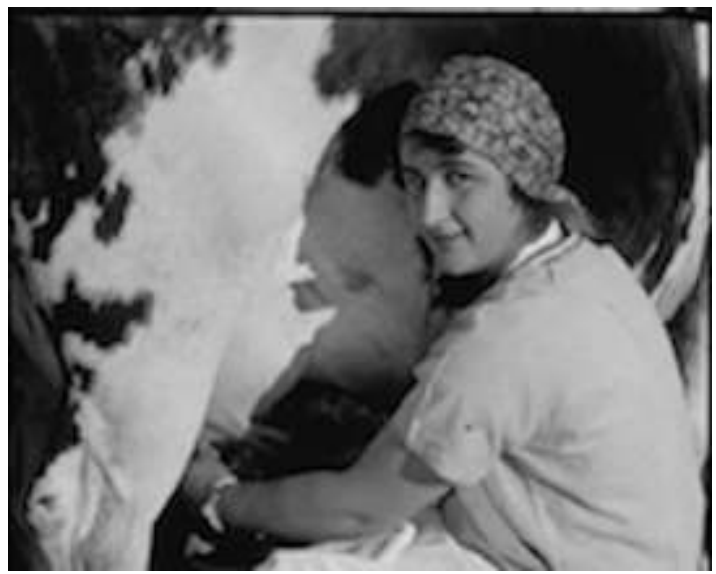

L'écossage et la traite dans Le Centre d'enseignement agricole et ménager de Coëtlogon-Rennes (Jean Benoit-Lévy, 1929)

Source : AFF - CNC

Concernant les films d'orientation professionnelle, le documentaire sur Le Centre d'enseignement agricole et ménager de Coëtlogon-Rennes (1929) est emblématique du rôle assigné aux femmes. Dans ce genre de films, il s'agit principalement d'éduquer les jeunes femmes à l'hygiène du foyer et à l'économie domestique. Diffusant le stéréotype de la femme cantonnée au travail ménager, ils reprennent à leur compte la conception conservatrice de la gardienne du foyer, dévouée à la maternité et aux tâches ménagères, telle qu'elle est communément admise à l'époque dans les films d'orientation professionnelle. Cette conception transparaît aussi dans de nombreux films hygiénistes, notamment La Contagion par les crachats (1925), La Contagion par la poussière (1925), La Bonne Voisine (1925), Le Nid (1928), ou encore L'Ange du foyer (1928). Dans les cartons (ou intertitres) de ces films, cette vision conservatrice est paradoxalement présentée comme moderne sous prétexte que les femmes apprennent à utiliser des outils ménagers « dernier cri ».

7 Cependant, ces films témoignent d'une conception moins conservatrice qu'il n'y paraît en faisant la promotion des nouveaux centres de formation. Ils mettent l'accent sur la nouveauté de l'enseignement qui est proposé dans les établissements publics, qui se trouvent en concurrence avec les écoles ménagères catholiques, largement majoritaires dans la formation des femmes. Tandis que l'enseignement privé est présenté comme archaïque, les films d'orientation professionnelle laïques mettent en exergue la modernité des contenus enseignés, non pas seulement dans le domaine professionnel, mais aussi dans l'enseignement général. Par exemple, le film sur Le Centre d'enseignement agricole et ménager de Coëtlogon-Rennes (1929) met en exergue la qualité des « enseignements théoriques par des cours et des conférences ».

8 Néanmoins, les orientations professionnelles, liées aux types de métiers enseignés dans ces écoles, participent toujours de la même distinction genrée entre métiers d'hommes (ferronnerie d'art, chaudronnerie, ébénisterie) et métiers de femmes (couturière, lingère, brodeuse, fourreuse), en particulier dans Doigts d'ouvrières, mains de fées (1925) et dans Des métiers pour les jeunes filles (1928) ${ }^{3}$. 
Images 3 et 4.

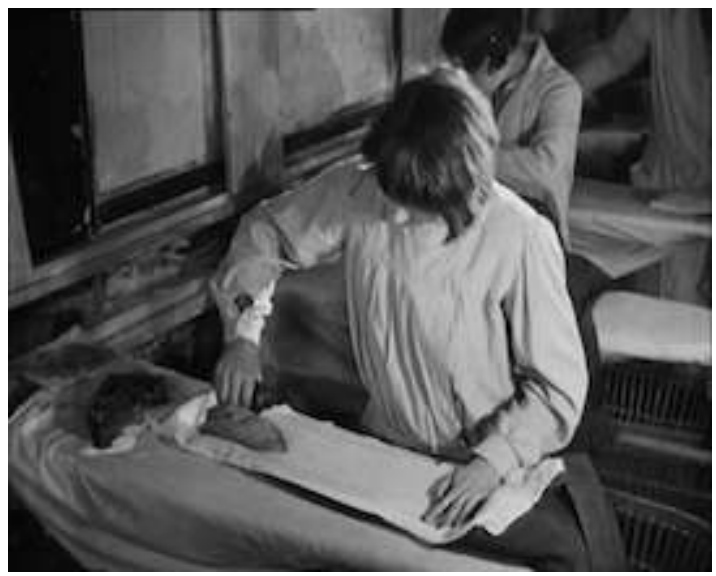

Image 4.

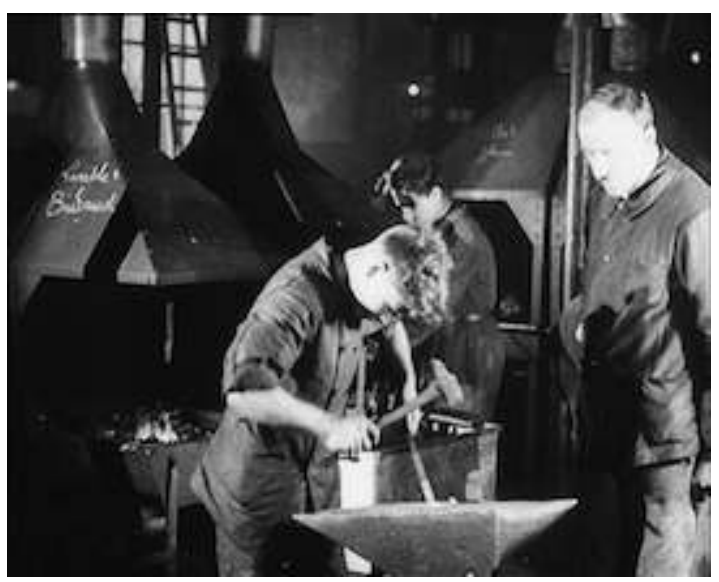

La lingerie et la ferronnerie dans Des métiers pour les jeunes filles (Jean Benoit-Lévy, 1928) et Des métiers pour les jeunes gens - Des métiers masculins (Jean Benoit-Lévy, 1928)

Source : AFF - CNC

En matière de représentation, les films d'orientation professionnelle obéissent aux lois des films institutionnels ou films d'entreprise, autrement appelés «films utilitaires » (« utility films», Hediger et Vonderau, 2009). Parce qu'ils ont un commanditaire institutionnel, en l'occurrence le ministère de l'Instruction publique ou le ministère de l'Agriculture, ils ne font qu'encenser les dispositifs de formation, qui sont systématiquement présentés de manière euphorique. À ces films muets, il ne manque que le son pour donner la vision idyllique du travail en chantant. Qu'ils soient destinés aux filles ou aux garçons, les motifs dénotant la joie d'apprendre un métier y sont nombreux: place importante accordée au filmage des temps de récréation, mise en scène de rondes, par exemple. Autant dire que ces films de propagande (le mot n'est pas péjoratif à l'époque) sont d'abord des outils de communication avant de constituer un témoignage sur le travail féminin. 
Images 5,6 et 7 .

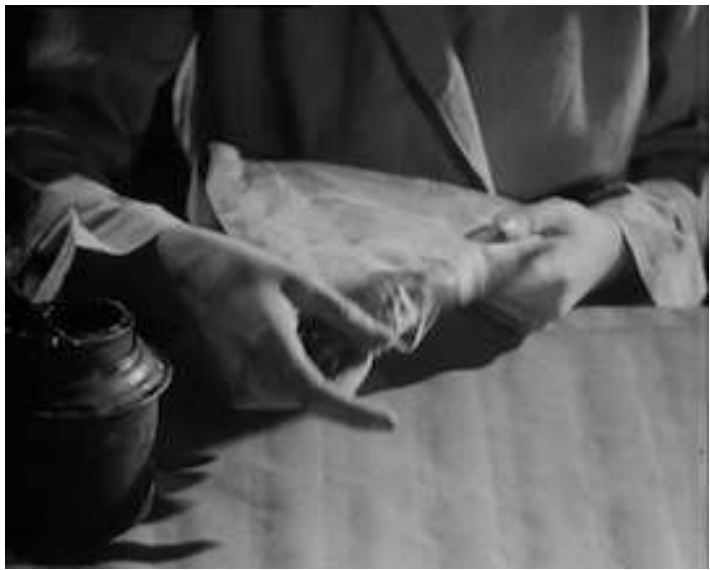

Image 6.

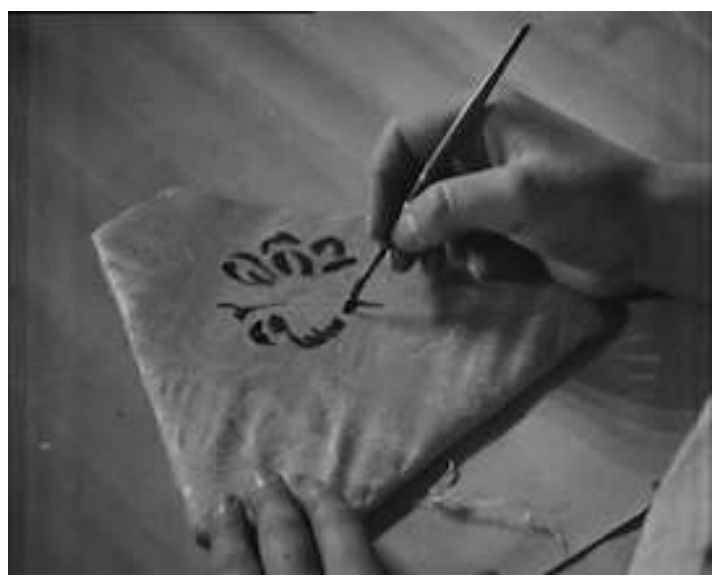

Image 7.

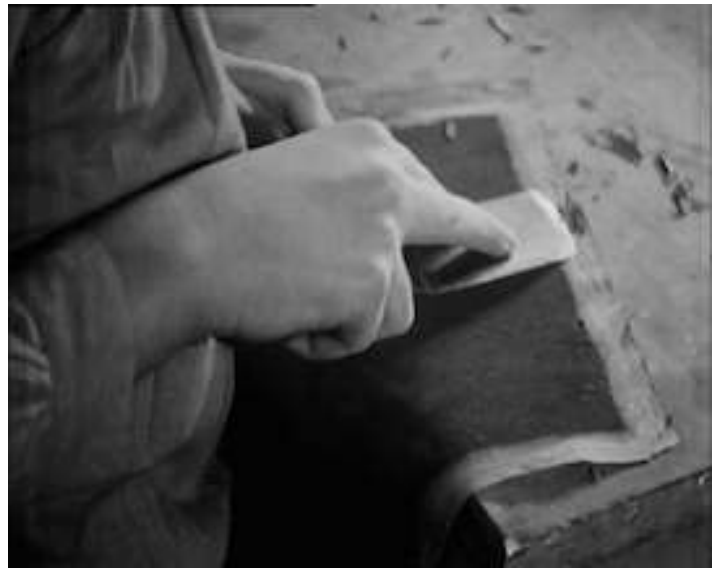

Mains d'ouvrière en papeterie et maroquinerie dans Des métiers pour les jeunes filles (Jean BenoitLévy, 1928)

Source : AFF - CNC

10 Cependant il est possible d'y déceler, dans le choix de certains cadrages, la place assignée aux femmes, qui sont orientées vers des métiers où la dextérité et la minutie sont requises, par exemple dans la couture ou encore la papeterie. Doigts de fée... Mains 
d'ouvrière (1925) et Des métiers pour les jeunes filles (1928) sont exemplaires de cette forme de discrimination prétendument fondée sur des raisons morphologiques, car ils accumulent un nombre de gros plans sur la partie de l'anatomie des travailleuses qui intéresse le plus le réalisateur : la main.

Images 8, 9 et 10 .

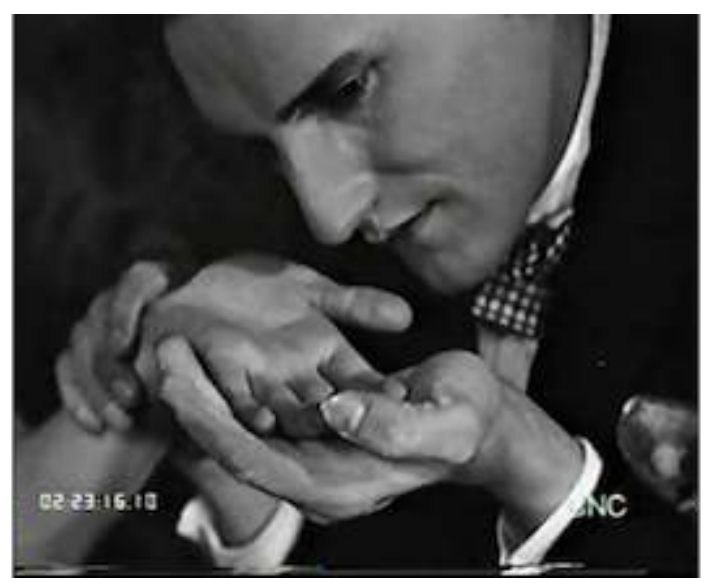

Image 9.

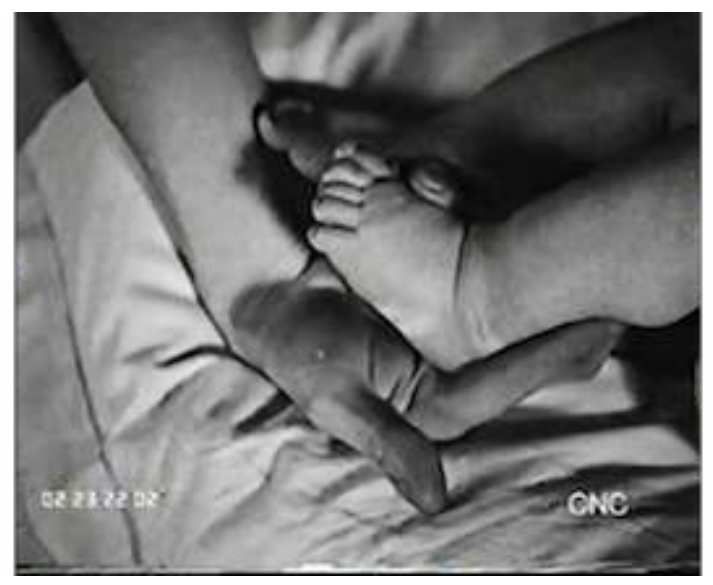


Image 10.

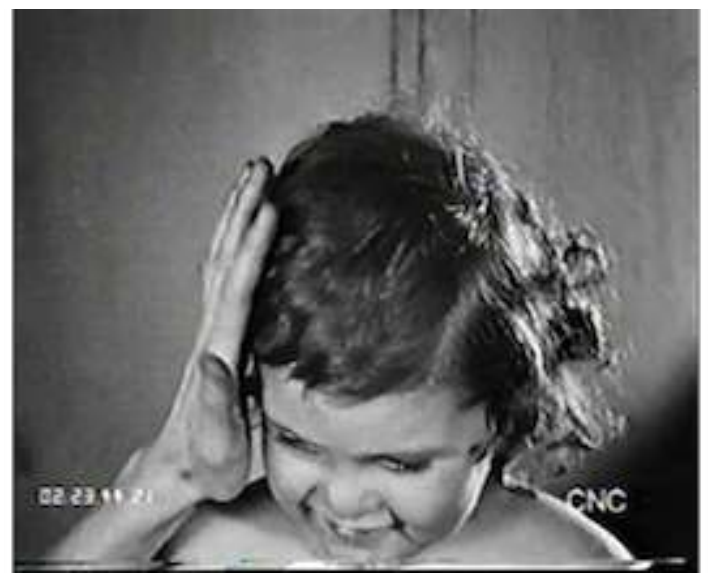

La main de l'infirmière vue par l'instituteur dans Le Voile sacré (Jean Benoit-Lévy, 1926)

Source : AFF - CNC

Si ces gros plans sur des mains féminines sont d'abord motivés par le souci de filmer des gestes professionnels, ils peuvent également présenter un caractère fantasmatique car ils sont récurrents dans les fictions du cinéaste. Probablement ses documentaires lui auront servi de bouts d'essai pour imaginer la fin du Voile sacré (1926), en tout point édifiante en matière de propagande nataliste. En effet, la romance entre la protagoniste principale, Margot Fréville (une infirmière puéricultrice), et Monsieur Cozette (un instituteur) prend fin dans une séquence qui laisse libre court à l'imagination de cet homme sur la main de cette femme. En l'espèce, Monsieur Cozette vient prendre le café chez Margot Fréville. Parce qu'il s'est endimanché, nous devinons son intention de lui demander sa main. Alors que la jeune femme s'adonne à la couture, l'instituteur lui saisit la main et récite un poème : «Douces mains...». Puis une suite de plans montés en accolade montre les mains de Margot s'occupant de tous les nourrissons dont elle a pris soin durant le film. Après une ellipse, nous comprenons que Margot, devenue Madame Cozette, connaît à son tour les joies de la maternité.

\section{Les métiers du soin dans les récits à visée éducative : La Future Maman et Le Voile sacré}

Outre les métiers manuels à connotation domestique auxquels sont généralement cantonnées les femmes, de nombreux films de Jean Benoit-Lévy diffusés dans les réseaux d'éducation populaire laïque représentent des métiers de service dans les domaines de la santé ou de l'éducation. Par là même, ils s'inscrivent dans l'idéologie du modèle de société prôné sous la IIIe République, qui met en exergue la promotion des filles d'agriculteurs au rang d'infirmière ou d'institutrice. 
Image 11.

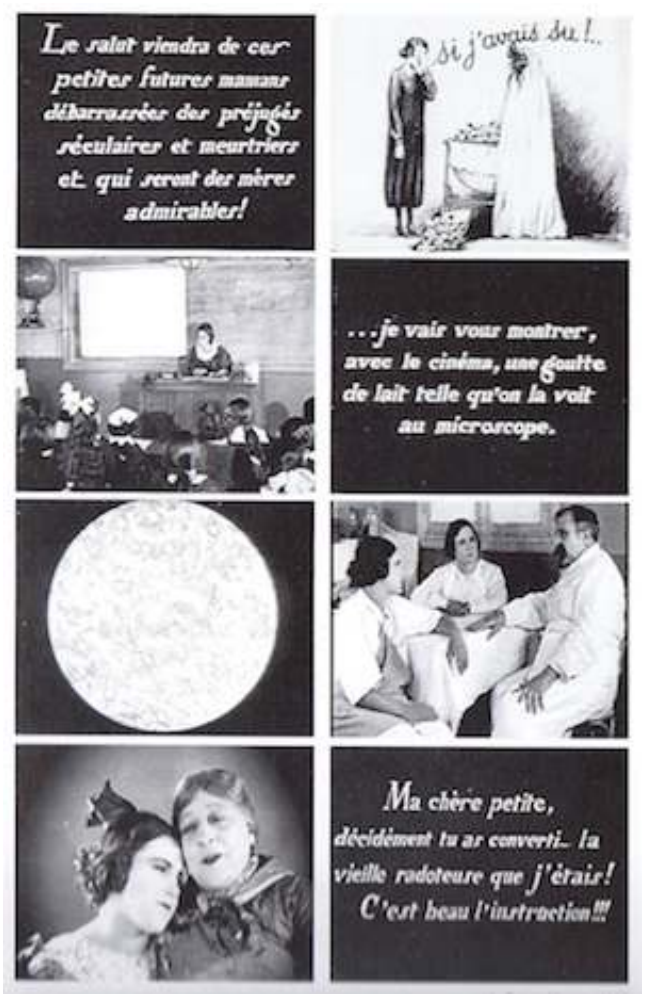

La jeune Margot prend modèle sur l'institutrice et l'infirmière dans La Future Maman (Jean Benoit-Lévy, 1924)

Source: AFF - CNC

Dans cette perspective, le personnage récurrent de Margot Fréville, fille de ferme promue infirmière visiteuse, est emblématique de tout le cinéma d'éducation populaire laïque de la IIIe République. Dans La Future Maman ${ }^{4}$, Margot est une jeune paysanne qui découvre la puériculture pour finalement réussir le concours d'infirmière dans Le Voile sacrés. Ces deux longs métrages d'éducation pour adultes se présentent comme des récits à valeur didactique où les histoires sont destinées à illustrer des contenus éducatifs en matière de puériculture, d'hygiène, de lutte anti-tuberculose et de prophylaxie $^{6}$. De fait, ce ne sont pas des documentaires mais des fictions très documentées. En effet, Marie Epstein ${ }^{7}$, qui travaillait aux scénarios, aimait ancrer ses récits dans le réel. Aussi, nous pouvons y trouver de nombreuses scènes où le travail des femmes est central. Fréquemment, les infirmières assistent un médecin qui occupe une position décisionnelle en raison de son statut, mais aussi dominante et charismatique, tel que le suggère le traitement filmique : condamnation sans appel des comportements à risque des mères; froncement de sourcils accompagnant des répliques dites sur un ton péremptoire.

Si le médecin vilipende les patients irresponsables, il n'est pas plus tendre avec les infirmières. Ce rapport de travail fortement dissymétrique est montré dans une séquence où Margot Fréville passe l'examen d'infirmière devant un jury constitué de vieux professeurs à l'air inquisiteur, sous le regard inquiet de Madame Mabu, une femme qui l'accompagne. Cet examen n'est pas représenté selon une esthétique réaliste, mais tel que Madame Mabu voit les examinateurs (en occularisation interne): 
des lentilles déformantes sont ainsi utilisées pour rendre les médecins à la fois terrifiants et grotesques, comme dans un film d'avant-garde des années 1920.'

Images 12, 13 et 14.

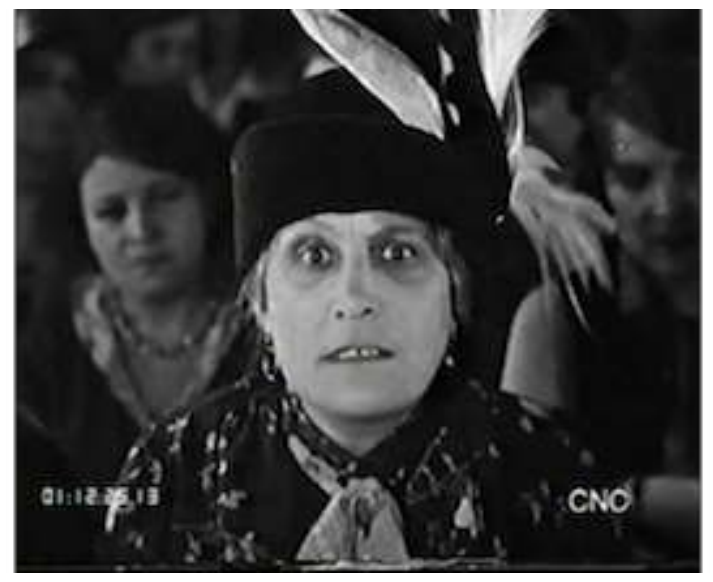

Image 13.

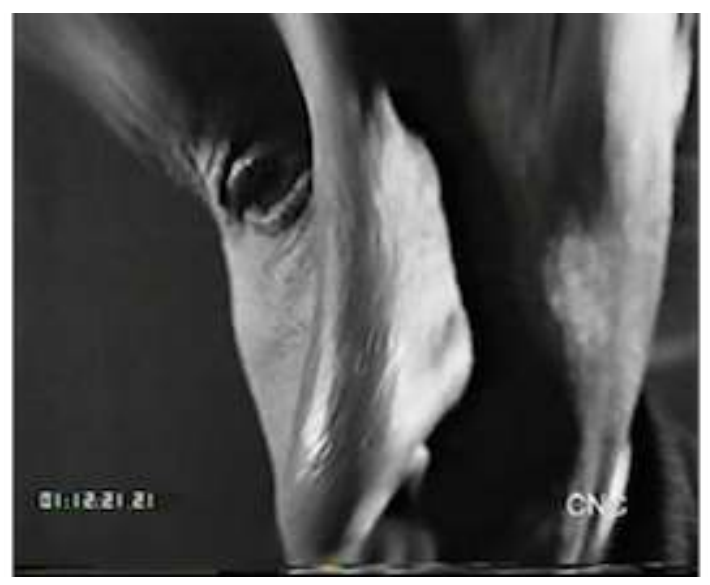

Image 14.

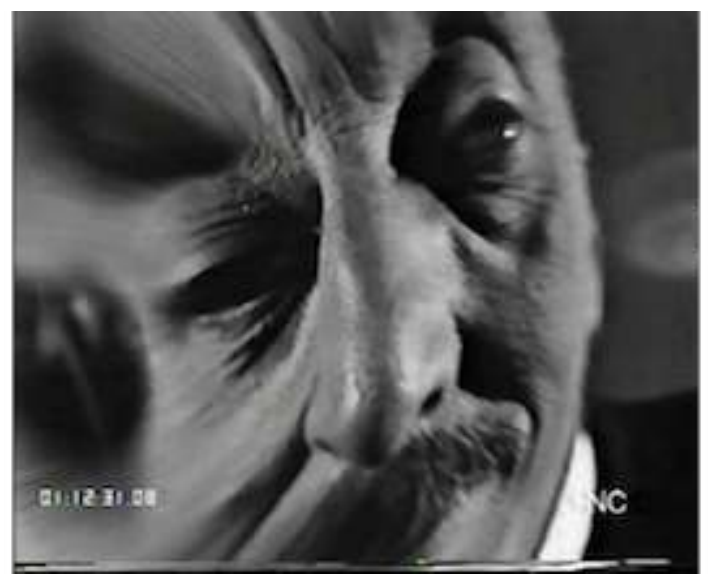

Le jury vu par Madame Mabu dans Le Voile sacré (Jean Benoit-Lévy, 1926) Source : AFF - CNC 
métrages, lui permettent-ils de développer un discours plus complexe sur la condition féminine. Si ces films reprennent les archétypes de l'orpheline, de la femme isolée, de la prostituée si populaires dans le mélodrame, c'est pour mieux dénoncer ces tabous et prôner l'émancipation des femmes des fléaux sociaux et du cadre patriarcal (Laborderie, 2010).

\section{Image 15.}

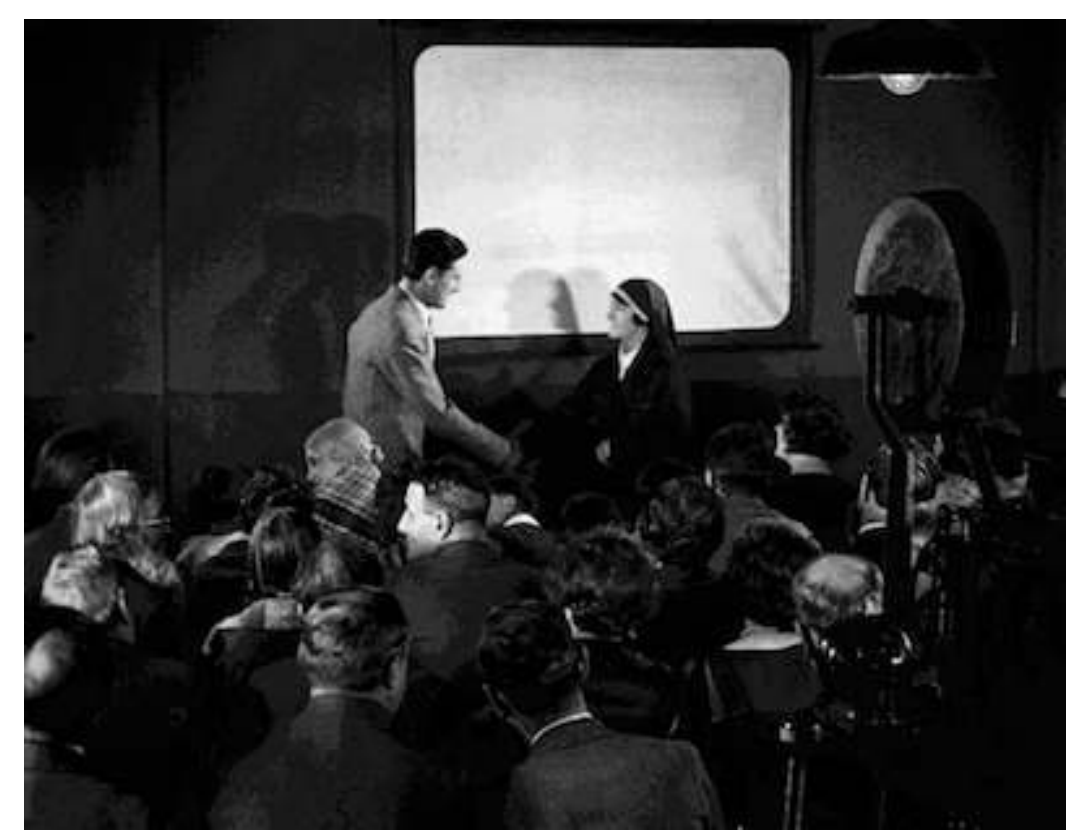

L'infirmière se marie avec l'instituteur dans Le Voile sacré (Jean Benoit-Lévy, 1926) Source : AFF - CNC

\section{La Maternelle, un plaidoyer pour l'émancipation des femmes}

En 1932, Jean Benoit-Lévy franchit une nouvelle étape en adaptant librement La Maternelle $^{8}$, roman de Léon Frapié, en collaboration avec Marie Epstein. Concernant la critique des rapports femmes/hommes au travail, ce film atteint un nouveau palier car il reprend l'ensemble des stéréotypes de genre présents dans les précédents films pour mieux les critiquer.

18 Il se présente tout d'abord comme un plaidoyer en faveur de la protection de la petite enfance et s'inscrit par là même dans la lignée des films d'éducation populaire qui ont fait le succès de Jean Benoit-Lévy dans les réseaux du cinéma éducateur laïque. En même temps, il occupe une place centrale dans l'œuvre du réalisateur, puisqu'il 
récapitule les thèmes et la forme des films d'éducation tout en se tournant vers le cinéma commercial. En tant qu'adaptation libre, le film fait l'apologie des écoles maternelles et donne la part belle au personnage de Rose, une aide maternelle qui réussit à s'émanciper par son travail (tandis que le roman est féroce envers le système scolaire et profondément misogyne).

En résumé, Rose, une jeune fille de bonne famille, se retrouve sans ressources à la suite de la mort de son père et de l'abandon de son fiancé. Malgré le fait qu'elle soit titulaire d'un baccalauréat, elle est contrainte de prendre dans la précipitation un emploi de femme de service dans une école maternelle. Ce recrutement est tout d'abord mal accueilli par le docteur Libois, le médecin de la maternelle. L'administration a en effet nommé Rose par erreur à la place de la protégée du docteur, dont nous comprenons qu'il entend imposer ses vues sur le recrutement du personnel de la maternelle. Cela n'empêche pas la jeune femme de prendre goût à son travail. Découvrant les difficultés sociales auxquelles les enfants sont confrontés, elle recueille chez elle la petite Marie, qui a été abandonnée par sa mère. À la fin du film, la directrice de la maternelle propose à Rose un emploi d'institutrice dans une école éloignée, ce qui aurait pour conséquence de la séparer de la petite fille. Au même moment, le docteur, qui s'est épris de la jeune femme, demande sa main, à condition qu'elle renonce à son métier de fille de service, ce qui suscite l'inquiétude de Marie. L'enfant, qui croit être une nouvelle fois abandonnée, fugue le long de la Seine, tombe à l'eau, mais est finalement sauvée. Rose préfère refuser le mariage et l'emploi d'institutrice, plutôt que d'abandonner la petite fille. Cette décision force l'admiration du docteur et de la directrice, qui acceptent finalement les raisons de Rose. La jeune femme pourra demeurer à la maternelle et épouser le docteur, qui finit par adopter la petite Marie.

Le film fait ainsi la critique d'au moins deux rapports de domination homme/femme au travail :

1. Une femme ne peut pas exercer une profession à responsabilité sans la caution d'un homme.

2. Malgré leurs diplômes, les femmes doivent consentir à un métier subalterne. En l'occurrence, Rose est abandonnée au début du film par son fiancé, ce qui fait d'elle une femme isolée. À la recherche d'un emploi pour assurer sa subsistance, elle ne bénéficie d'aucun appui masculin ce qui la contraint d'accepter un poste de fille de service, alors même que son baccalauréat peut la laisser prétendre à un poste d'institutrice.

21 Dans la séquence de la demande en mariage, la dissymétrie de la relation entre Rose et le docteur Libois est très bien soulignée par la mise en scène qui montre Rose en plongée, comme soumise au regard du médecin. De surcroît, le docteur s'affranchit des codes sociaux en s'asseyant sur la table et enserre les mains de la jeune fille de service, autant de signes qui marquent la tentative de domination masculine. 
Images 16 et 17 .

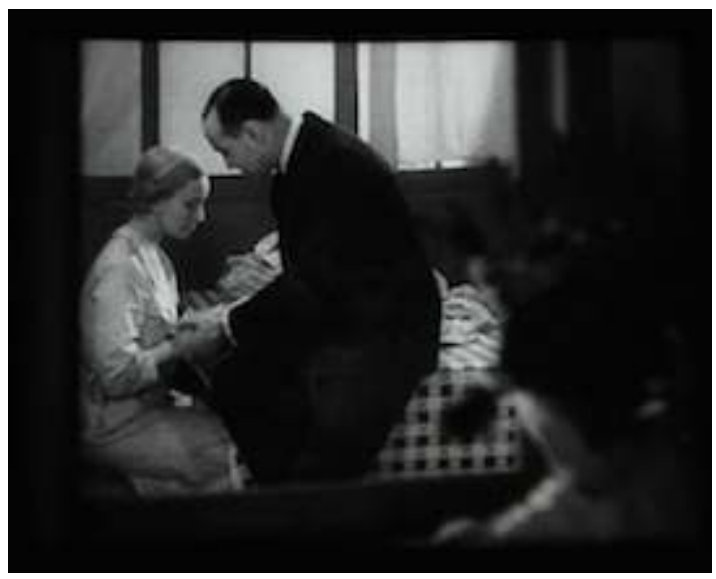

Image 17.

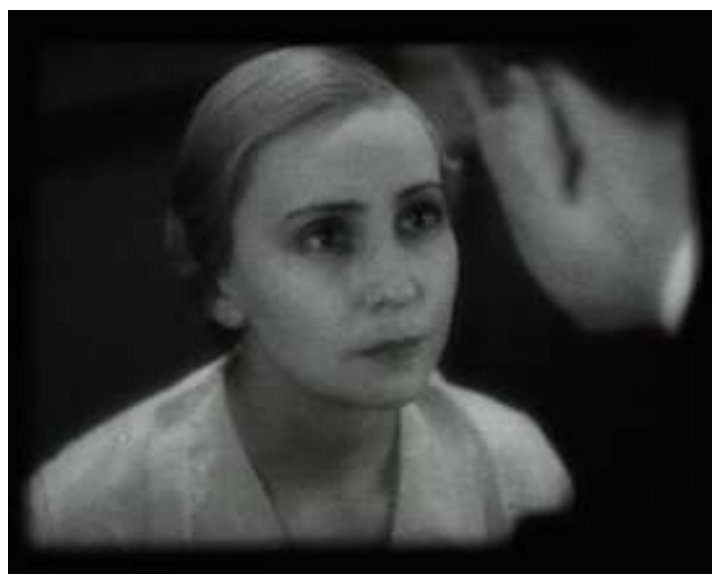

Le regard dominateur du docteur Libois dans La Maternelle (Jean Benoit-Lévy, 1932)

Source : AFF - CNC et Archives privées de Liliane Jolivet

Ce travail sur les connotations filmiques est bien la marque d'un discours intentionnel sur les rapports femmes/hommes. De la même manière, Jean Benoit-Lévy critique le fait que la prise de parole, en tant que signe de pouvoir, appartienne exclusivement aux hommes. Au début du film, Rose est en effet subjuguée par la parole masculine, précisément par une promesse de mariage qui ne sera pas tenue. À la fin du film, elle demeure, dans un premier temps, muette devant la proposition d'un beau mariage et d'un poste à responsabilité. Puis, contre toute attente (et contrairement au personnage de Margot Fréville), elle refuse la belle position sociale qui lui est promise sous le couvert d'un homme. Finalement, le docteur Libois doit lui concéder l'adoption de la petite Marie et la conservation de son poste à la maternelle. Aussi, la réplique leitmotiv du film, «Marie, tiens-toi droite », que Rose répète à la petite enfant pour soigner son dos atteint d'une scoliose, devient en contexte un symbole d'émancipation, qui sonne comme un appel aux femmes à relever la tête face aux prétentions dominatrices des hommes 9 .

La Maternelle revêt enfin une autre dimension politique en prônant une plus grande mixité sexuée et sociale à l'école. Rappelons d'abord qu'au moment où il est réalisé, la maternelle est la seule école où la mixité sexuée est acceptée, puisque la gémination ne 
sera légalisée à l'école primaire qu'en 1933. Jean Benoit-Lévy montre ainsi la maternelle comme un lieu ouvert à une mixité, dont le filmage documentaire d'une petite fille noire présente tout au long du film devient le symbole. Aussi, au début des années 1930, la maternelle, elle-même héritière des salles d'asile, demeure une école des pauvres plutôt qu'une école pour tous. D'une certaine manière, ce rôle d'assistance et de protection l'empêche d'évoluer vers des missions d'éducation seules susceptibles de favoriser la promotion sociale. Le film est ainsi porteur d'un discours ambitieux en matière de réussite sociale, en particulier lorsqu'il milite contre l'étanchéité des classes sociales. Dans cette perspective, l'impossibilité de travailler à la maternelle pour une jeune femme issue de la bourgeoisie est montrée comme une limite du système. Dans le contexte de l'entre-deux-guerres, les écoles publiques maternelles et primaires sont, en effet, animées par des normaliennes généralement issues du peuple. Rose, issue d'un milieu bourgeois et possédant son brevet supérieur, a toutes les peines du monde à se faire accepter par les divers personnels de la maternelle. Loin de présenter les quatre murs de la classe comme les murs d'une prison, comme le faisait le roman dont le film est l'adaptation, La Maternelle ne décrit pas l'école comme un espace d'enfermement, mais plutôt de protection et d'émancipation. Pour autant le film pointe cette prison plus invisible quant à elle, celle que forment les classes sociales et dont l'école éprouve des difficultés à s'affranchir. Si l'école laïque a derrière elle cinquante ans d'existence, une conception élargie de la notion de laïcité la porte en ce début des années 1930 sur le terrain social. La Maternelle de Jean Benoit-Lévy annonce ainsi le renouveau du projet d'éducation nationale dont le Front populaire sera porteur.

\section{Un engagement aux motivations multiples}

Pour quelles raisons Jean Benoit-Lévy attache-t-il tant d'importance à la place des femmes dans la société? Nous voyons au moins trois motivations biographique, sociopolitique et artistique. Tout d'abord, il ne faut pas exclure une raison autobiographique: Jean Benoit-Lévy devient père de deux petites filles au moment même où il entreprend la réalisation de ces premiers films mettant en exergue le travail des femmes. Selon des témoignages de son entourage familial, notamment d'une de ses petites-filles, Liliane Jolivet, il s'attacha à donner à ses filles une "éducation moderne » en tenant par exemple à ce qu'elles reçoivent une éducation à la sexualité, trait relativement atypique, à l'époque, dans l'éducation des filles ${ }^{10}$.

Par ailleurs, le cinéma de Jean Benoit-Lévy, très documenté et ancré dans le réel, se veut en phase avec la réalité sociopolitique de son époque. Dans cette perspective, l'opposition ville/campagne est prégnante dans tous les films de Jean Benoit-Lévy, en particulier dans La Future Maman (1924) et Le Voile sacré (1926). Ces films font allusion au problème de l'exode rural, notamment des femmes qui vont chercher un travail à la ville. Dans le cinéma de l'entre-deux-guerres, selon un stéréotype courant, la ville est généralement représentée comme la source de tous les maux, tandis que la campagne est censée sauver la société de l'anéantissement. Cependant, les films de Jean BenoitLévy échappent, au moins partiellement, à ce manichéisme : aussi bien la ville que la campagne ont besoin l'une de l'autre. Le personnage de Margot Fréville, d'abord migrant à la ville, puis revenant à la campagne est emblématique de ce rapport complexe entre les deux espaces. 

femmes dans le monde ouvrier agricole. Selon un mouvement amorcé au début du XXe siècle (et accéléré durant la Première Guerre mondiale), le nombre de femmes chefs d'exploitation agricole ou ouvrières agricoles ne cesse d'augmenter sur la période. Dans le même temps, les femmes migrant à la ville pour occuper un travail rémunéré augmente fortement ${ }^{11}$. Si d'un côté, selon une vision conservatrice et patriarcale, les paysannes sont toujours censées retenir les paysans sur leurs terres en s'occupant de leurs foyers, tel qu'on l'enseigne dans les écoles ménagères privées, d'autres voies leur sont maintenant ouvertes, notamment celles des métiers industriels, de l'enseignement ou du secteur sanitaire et social. Toujours en phase avec la réalité sociopolitique, les films de Jean Benoit-Lévy s'inscrivent dans le droit fil de la politique (volontariste et/ou utilitariste) de l'État en matière d'éducation des filles avec l'ouverture aux femmes de filières professionnelles industrielles durant la première moitié du XXe siècle. De manière moins consensuelle et plus orientée politiquement, les films de Jean BenoitLévy revendiquent une certaine évolution du rôle des femmes dans la société française. Ils témoignent d'une évolution d'un discours nataliste communément admis (en raison notamment du grave déficit démographique dû à la Première Guerre mondiale) vers une conception plus progressiste et se conforment en cela à l'idéal d'émancipation que prônent les radicaux et, dans une plus large mesure, les socialistes. l'importance des femmes dans les productions de Jean Benoit-Lévy. Sans doute le choix du genre mélodramatique l'explique-t-il en partie. Par ailleurs, nous ne pouvons pas négliger l'influence du cinéma soviétique dont Jean Benoit-Lévy était admirateur : nous pensons, par exemple, à la glorification du travail féminin dans La Ligne générale de Sergueï Eisenstein et Grigori Alexsandrov (1929). Mais il faut surtout souligner le rôle majeur joué par Marie Epstein dans la conception des films de Jean-Benoit-Lévy. Cette collaboration était si étroite, que Jean Benoit-Lévy l'avait inscrite au générique, en tant que scénariste, assistante-réalisatrice et même coréalisatrice, dans onze de ses longs métrages, si bien qu'un certain nombre d'études féministes ont même fini par considérer de manière excessive que des films, tels que La Maternelle, avaient été exclusivement réalisés par Marie Epstein sans le concours de Jean Benoit-Lévy (Flitterman, 1990) ${ }^{12}$.

\section{Conclusion}

En définitive, la représentation du travail féminin occupe une place prépondérante dans la cinématographie de Jean Benoit-Lévy, qui constitue en cela une source non négligeable sur la construction, puis sur la critique des stéréotypes de sexe sous la IIIe République. Malgré l'hétérogénéité des genres de films étudiés (films d'orientation professionnelle, films éducatifs pour adultes, fictions tout public), malgré le temps qui sépare ses premiers courts métrages muets des grandes fictions distribuées à l'international, il est possible d'y déceler l'élaboration d'un discours critique sur le rôle des femmes dans la société. Mise à part la vision du cinéaste, ces films reflètent surtout les évolutions socioéconomiques de l'entre-deux-guerres en France ainsi que l'idéologie des gauches fréquemment au pouvoir sous la IIIe République, dont Jean Benoit-Lévy est le propagandiste le plus important. 
asant la place au foyer que les hommes veulent leur assigner, les personnages féminins des fictions de Jean Benoit-Lévy, à l'instar de Rose dans La Maternelle, sont fréquemment des femmes célibataires, qui préfèrent se consacrer à leur vie professionnelle plutôt que familiale. Si cette vision peut être considérée comme relativement progressiste dans le contexte historique et notamment dans le nuancier politique de l'entre-deux-guerres (rappelons que le programme familial de Vichy s'est constitué en réaction aux politiques sociales des Cartels des gauches et du Front populaire), un point de vue plus féministe pourrait y voir au moins deux limites : d'une part, les métiers dits « féminins » demeurent des métiers subalternes sous la coupe de métiers masculins (comme dans le cas du tandem infirmière / médecin) ; d'autre part, la vie professionnelle des femmes n'est possible que si elle est consentie par leurs chefs de service et leurs maris. Il est ainsi possible d'y voir une forme de sexisme bienveillant dans la mesure où les hommes gardent le dernier mot, tout en faisant figure de compagnons éclairés.

\section{BIBLIOGRAPHIE}

Duby G. (1977), Histoire de la France rurale, Tome 4. La fin de la France rurale de 1914 à nos jours, Paris, Seuil.

Flitterman-Lewis S. (1990), To Desire Differently: Feminism \& the French Cinema, Sandy FlittermanLewis, Urbana: University of Illinois.

Hediger V. et Vonderau P. (2009), Films that Work: Industrial Film and the Productivity of Media, Amsterdam, Amsterdam University Press.

Laborderie P. (2009), « Le film-parabole dans les Offices du "cinéma éducateur" en France dans l'entre-deux-guerres (histoire d'un cinéma de propagande et étude d'un genre cinématographique) ", thèse de doctorat en études cinématographiques et audiovisuelles, dir. Roger Odin et Michel Marie, Université Sorbonne nouvelle - Paris 3.

Laborderie P. (2010), « Le Voile sacré (Jean Benoit-Lévy, 1926), un film d'éducation populaire dans le réseau du cinéma éducateur laïque », in F. Laot F. (dir.), L'image dans l'histoire de la formation des adultes, Paris, L'Harmattan, p. 31-48.

Laborderie P. (2013), « Les stéréotypes de la fugue et de la rébellion dans la Maternelle (Jean Benoit-Lévy et Marie Epstein, 1932) », Cahiers Robinson, n³3, p. 15-30.

Laborderie P. (2015), Le cinéma éducateur laïque, Paris, L'Harmattan.

Sauvy A. (1965), Histoire économique de la France entre les deux guerres, Paris, Fayard ; Paris, Economica, 1984.

Sellier G. (1999), « Le cinéma français des années trente » in Christine Bard (dir.), L'Antiféminisme au XXe siècle, Paris, Fayard.

Vignaux V. (2007), Jean Benoit-Lévy ou le corps comme utopie, une histoire du cinéma éducateur dans l'entre-deux-guerres en France, Paris, AFRHC. 
Vignaux V. (2008), « Un cinéma éducateur dit de "propagande sociale” dans l'entre-deux-guerres en France ou Des images pour la République », in Bertin-Maghit J.-P. (dir.), Une histoire mondiale des cinémas de propagande, Paris, Nouveau Monde éditions, p. 199-213.

\title{
Filmographie indicative des films de Jean Benoit-Lévy sur les métiers
}

\author{
Contagion par les crachats $(1925,1$ min $53 \mathrm{~s})$ \\ Des métiers pour les filles (1928, $11 \mathrm{~min})$ \\ Des métiers pour les jeunes filles (1928, $18 \mathrm{~min})$ \\ Des métiers pour les jeunes gens - Des métiers masculins (1928, 30 min) \\ Doigts d'ouvrières, mains de fées (1925, 24 min) \\ Hélène (1936, $101 \mathrm{~min})$ \\ Histoire d'un chapeau de paille : la modiste (1925, court) \\ Itto $(1935,117 \mathrm{~min})$ \\ La Bonne Voisine (1925, 3 min $15 \mathrm{~s})$ \\ La Contagion par la poussière (1925, 2 min $15 \mathrm{~s})$ \\ La Ferronnerie d'art (1924, court) \\ La Future Maman (1924, 60 min) \\ La Maternelle (1932, $83 \mathrm{~min})$ \\ La Mort du cygne (1937, $100 \mathrm{~min})$ \\ L'Ange du foyer (1928, $35 \mathrm{~min})$ \\ Le Centre d'enseignement agricole et ménager de Coëtlogon-Rennes (1929, 24 min) \\ Le Nid (1928, 26 min) \\ Le Voile sacré (1926, $81 \mathrm{~min})$ \\ Petits doigts de fée : la lingerie (1925, $22 \mathrm{~min})$ \\ Un beau métier méconnu : le repassage (1925, court)
}

\section{NOTES}

1. L'UFOCEL est plus connue sous le nom qu'elle prit en d'Union française des œuvres laïques d'éducation par l'image et le son (UFOLEIS).

2. Nous livrons une filmographie sélective en annexe. Pour une vision complète du cinéma de Jean Benoit-Lévy, voir l'ouvrage que Valérie Vignaux a consacré au cinéaste (Vignaux, 2007) ainsi que le livre issu de notre thèse (Laborderie, 2015).

3. Films visionnés à la cinémathèque municipale de Saint-Étienne: la Ferronnerie d'art (Jean Benoit-Lévy, 1924) ; Histoire d'un chapeau de paille : la modiste (Jean Benoit-Lévy, 1925) ; Un beau métier méconnu : le repassage (Jean Benoit-Lévy, 1925) ; Petits doigts de fée : la lingerie (Jean BenoitLévy, 1925) ; Des métiers pour les jeunes gens - Des métiers masculins (Jean Benoit-Lévy, 1928) ; Des métiers pour les jeunes filles (Jean Benoit-Lévy, 1928). 
4. Fiche technique : La future Maman; France ; 1924 ; éRalisation : Jean Benît-Lévy ; Scénario : Dr Louis Devraigne; Opérateur : Edmond Floury; Production : EFC/Films ; Dessins animés : Albert Mourlan; Distribution: Mademoiselle Odette (un bébé) ; éLone Balme (Margot, fille de ferme, sœur du bébé) ; Madame Decori (La mère Mabu) ; Madame Lejeune (fermière, mère de Margot) ; Monsieur Duribert (fermier, père de Margot) ; Noir et blanc ; Muet ; Durée : $60 \mathrm{mn}$; film visionné aux Archives française du film du C.N.C

5. Le Voile sacré; France ; 1926 ; Réalisation: Jean Benoit-Lévy ; Scénario: Dr Louis Devraigne ; Opérateur: P. Cotheret; Production: l'Office national d'hygiène sociale et le Pari mutuel ; Noir et blanc; Muet; Durée: $81 \mathrm{mn}$; Distribution des personnages: Margot Fréville (jeune visiteuse d'hygiène); Madame Mabu (épicière); docteur Rigal; Monsieur Cozette (instituteur). Le personnage de Madame Mabu est joué par Madame Decori. Film visionné aux Archives françaises du film du C.N.C.

Pour une analyse détaillée du film, voir Laborderie, 2010.

6. Aujourd'hui, ces films seraient sans doute classés dans les programmes comme des «docufictions ".

7. Cofondatrice en 1936 de la Cinémathèque française (aux côtés de son frère Jean Epstein, Henri Langlois et de Georges Franju), actrice, scénariste et réalisatrice, Marie Epstein est une figure marquante du cinéma français de l'entre-deux-guerres. Voir sa fiche biographique publiée sur Ciné-ressources : http://cinema.encyclopedie.personnalites.bifi.fr/index.php?pk=13654 Voir aussi « Marie Epstein: A Woman in the Shadows », Flitterman-Lewis, 1990, 141-168.

8. Fiche technique: La Maternelle; France; Production: 1932; Sortie: 1933; Scénariste et réalisateur : Jean Benoit-Lévy ; Assistant réalisateur : Marie Epstein ; Scénario d'après un roman de Léon Frapié ; Musique : Edouard Flament ; Photographie : Georges Asselin ; Son : Jean Dubuis ; Procédé sonore : Photosonor; Distributeur : Universal ; Distribution : Madeleine Renaud (Rose), Henri Debain (le docteur Libois), Mady Berry (Mme Paulin), Alice Tissot (la directrice), Sylvette Fillacier (M'âme Coeuret), Alex Bernard (le recteur), Paulette Élambert (Marie Coeuret) ; Noir et blanc; Sonore; Durée: 1 h $30 \mathrm{mn} 30 \mathrm{~s}$; Film conservé par les Archives françaises du film du Centre national de la cinématographie.

Pour une analyse détaillée du film, voir Laborderie 2013.

9. De fait, Rose constitue l'archétype de tous les personnages féminins qui suivront dans la cinématographie de Jean Benoit-Lévy. Ce sont toujours des femmes déterminées dans leur désir d'émancipation et dans leur travail. Ainsi de Itto, d'Hélène (dans les films éponymes datant de 1935 et 1936) et de Karine dans La Mort du cygne (1937). Il est à noter que cet idéal féminin est interprété à deux reprises par Madeleine Renaud dans La Maternelle et Hélène.

10. Pascal Laborderie, "Entretiens avec Liliane Jolivet », avril 2011.

11. Dans l'entre-deux-guerres, le monde paysan est important : en 1921, il représente environ cinq millions d'hommes et, en 1931, 4,36 millions, soit entre 36 et $42 \%$ de la population active (Duby, 1977, 82-87). Selon Alfred Sauvy, entre 1919 et 1931, l'exode rural a touché 9500000 personnes. En 1931, la population urbaine finit même par dépasser la population rurale (Sauvy, $1965 ; 1984,148$ ). On constate à la fois un fort exode rural des hommes et des femmes (pour des raisons économiques) et une augmentation à la campagne du travail des femmes.

12. Notre recherche dans les archives privées de Liliane Jolivet a montré qu'il n'en était rien. Il est possible d'y consulter un tapuscrit du scénario entièrement annoté de la main de Jean BenoitLévy. Sandy Flitterman elle-même est revenue sur sa position dans un article publié sur internet : https://en.wikipedia.org/wiki/La_Maternelle_(film). This page was last modified on 23 April 2015, at 16:40. Sandy Flitterman-Lewis, PhD. Associate Professor in English and Cinema Studies at Rutgers School of Arts and Sciences and author of To Desire Differently: Feminism \& the French Cinema, July 4th 2007, http://www.imdb.com/user/ur15920897/comments, accessed February 21, 2010. 


\section{RÉSUMÉS}

Dans l'entre-deux-guerres, la Ligue française de l'enseignement organisa un vaste réseau d'éducation populaire par le cinéma, «le cinéma éducateur laïque», qui se voulait éduquer à la santé et la citoyenneté les jeunes gens et les adultes. Ayant réalisé près de 350 films, Jean BenoitLévy est le réalisateur le plus emblématique de ce cinéma. Cet article étudie la représentation des métiers des personnages féminins qui peuplent sa cinématographie, aussi bien dans ses documentaires que dans ses fictions ancrées dans le réel. Si les premiers courts métrages de Jean Benoit-Lévy perpétuent les stéréotypes de genre (dans les films d'orientation professionnelle et les films hygiénistes), ses derniers longs métrages (par exemple, La Maternelle) témoignent d'une réflexion critique sur le rôle assigné aux femmes dans le travail et plus généralement dans la société.

Between the two World Wars, the French Educational League and the Regional Educational Cinema Offices, affiliated within the French Union of the Secular Educational Cinema Offices established a social use of a non-commercial cinema, the cinéma educateur. The educational cinema, with its after-school activities, was aimed at teens and adults in order to educate them to social hygiene and citizenship.

Jean Benoit-Levy, who directed almost 350 films (fiction and non-fiction, short or long), is the main leader of this republican centre-left propaganda. Generally speaking, his cinema campaigns for the evolution of the place of women in the French society in relationship to the practice of a job. Considering that getting a paid job can allow women to define their identity and fulfil themselves is a quite progressive idea at a time when in France women's right to vote is still in question.

\section{INDEX}

Mots-clés : cinéma, femmes, formation d'adultes, laïcité, métier, propagande

Keywords : adult education, cinema, labor, propaganda, secularism, women

\section{AUTEUR}

\section{PASCAL LABORDERIE}

Pascal Laborderie est docteur en Études cinématographiques et audiovisuelles de l'Université Sorbonne Nouvelle - Paris 3 et maître de conférences en Sciences de l'information et de la communication à l'Université de Reims Champagne-Ardenne (EA 4692 - CEREP). Historien de l'éducation populaire par le cinéma et sémiologue des films éducatifs, il a publié Le cinéma éducateur laïque (Paris, L'Harmattan, 2015, préface de Roger Odin) et coordonné un ouvrage intitulé La Ligue de l'enseignement et le cinéma, une histoire de l'éducation à l'image (F. GimelloMesplomb, P. Laborderie et L. Souillés-Debats dir., Paris, AFRHC, 2016). 